\title{
Aplicación de la metodología de aprendizaje experiencial en Educación Superior
}

\section{Application of the experiential learning methodology in Higher Education}

Verónica Alejandra Villarroel Henriquez ${ }^{1}$, María Pía Gutiérrez Suárez ${ }^{2}$, Daniela Valentina Bruna Jofré ${ }^{3}$,

INFORMACIÓN DEL

\section{ARTÍCULO}

Fecha de recepción: 16 de Julio de 2021.

Fecha de aceptación: 26 de Octubre de 2021.

Doctor en Psicología, Pontificia Universidad Católica de Chile. Docente-investigador, Universidad del Desarrollo - Chile.

E-mail: vvillarroel@udd.cl

Código ORCID:

https://orcid.org/0000-0002-3000-2248

${ }^{2}$ Magister en Psicología Clínica mención Psicoterapia Integrativa, Universidad Adolfo Ibáñez. Docente-investigador, Universidad del Desarrollo - Chile.

E-mail: mp.gutierrez@udd.cl

Código ORCID:

https://orcid.org/0000-0001-6948-278X

${ }^{3}$ Doctor en Psicología. Universidad de Concepción. Docente-investigador, Universidad del Desarrollo - Chile.

E-mail: dbrunaj@udd.cl

Código ORCID:

https://orcid.org/0000-0001-7424-2959

${ }^{4}$ Maestrante de Psychology of Education, University College of London. Docente-investigador, Universidad del Desarrollo - Chile

E-mail: icastillor@udd.cl

Código ORCID:

https://orcid.org/0000-0002-9941-1437

CITACIÓN: Villarroel, V., Gutiérrez, M.P., Bruna, D., \& Castillo, I. (2021). Aplicación de la metodología de aprendizaje experiencial en Educación Superior. Podium, 40, 41-58. doi:10.31095/podium.2021.40.3

\section{ENLACE DOI:}

http://dx.doi.org/10.31095/podium.202 1.40 .3

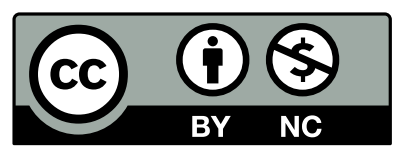

\section{Resumen}

Isidora Francisca Castillo Rabanal ${ }^{4}$

Se utilizó la metodología de aprendizaje experiencial en la asignatura de Psicología de la Adultez, de la carrera de psicología en una universidad chilena. El objetivo de este estudio de caso fue que los estudiantes pudieran aplicar y practicar sus conocimientos en una situación real y contextualizada, y que esto promoviera el logro de un aprendizaje profundo. La implementación se llevó a cabo por docentes y ayudantes en clases, ayudantías y actividades en terreno, en las que los estudiantes realizaron una variedad de actividades, entregando los productos correspondientes. La experiencia fue valorada por docentes y estudiantes, con efectos positivos en relación al desempeño de los estudiantes y a la forma en que se vincularon con el contexto y su futuro rol como psicólogos. Se concluye que el aprendizaje experiencial es una metodología de enseñanza valiosa, entregando directrices para incorporarla en el ámbito de la educación superior e introducir a los estudiantes a su rol profesional.

\section{Palabras Clave:}

Aprendizaje experiencial, educación superior, estudio de caso, psicología evolutiva, adultez, evaluación.

Clasificación JEL: 121, 123.

\begin{abstract}
An experiential learning methodology was used in the Psychology of Adulthood course, in the Psychology major of a Chilean university. The aim of this case study was for students to apply and put their knowledge into practice in a real, contextualized situation, and for this to impact on their in-depth learning. The implementation of this methodology was carried out by teachers and assistants during classes, tutoring and field trips, in which students had to execute a variety of activities and deliver a series of products. In general, positive results were observed regarding the students' performance and the way in which they engaged with the context and their future role as psychologists. In conclusion, experiential learning is a valuable tool, which offers guidelines for its implementation in Higher Education and introduce students to their future professional roles.
\end{abstract}

\section{Keywords:}

Experiential learning, higher education, case study, developmental psychology, adulthood, assessment.

JEL Classification: 121, 123.

\section{1}

PODIUM No. 40, Diciembre 2021, pp. 41-58

(C) Universidad Espíritu Santo - UEES

ISSN: 1390-5473 e-ISSN: 2588-0969 


\section{Introducción}

Ciertamente, la educación universitaria actual es diferente a la del siglo pasado en muchos aspectos. Uno de ellos es, el significativo aumento en el acceso a la educación superior, por lo que las características culturales y socioeconómicas de los estudiantes se han diversificado, y muchos de ellos son la primera generación de su familia que va a la universidad. El modelo tradicional de estudiante universitario que mostraba a un joven adolescente o adulto joven soltero, de nivel socioeconómico medio o alto, capaz de estudiar sin tener la necesidad de trabajar para satisfacer sus necesidades básicas $o$ pagar su educación, no representa a los miles de estudiantes que son la primera generación de universitarios en su familia. Tampoco a quienes se ven obligados a estudiar y trabajar, al mismo tiempo, a pesar de contar con becas y/o préstamos para movilizarse, alimentarse y pagar estudios, porque también deben apoyar económicamente a sus familias (Araneda-Guirriman y Pedraja-Rejas, 2017). El problema principal de hoy, radica en que muchos de estos nuevos estudiantes no cuentan con la preparación adecuada para la educación terciaria, siendo su capital cultural, social y económico menor en comparación con el estudiante universitario tradicional de treinta años atrás (Košutić, 2017).

Aún es posible encontrar brechas entre las competencias, conocimientos y preparación de los estudiantes que provienen de establecimientos públicos $\mathrm{y}$ particular-subvencionados, en comparación a los estudiantes de establecimientos privados. Ante esto, las instituciones de educación superior han debido cambiar el modelo educativo a nivel estratégico institucional y dentro del aula (Araneda-Guirriman y Pedraja-Rejas, 2017). Otros esfuerzos han incluido realizar talleres para que los estudiantes se adecúen al contexto universitario y la actualización de las competencias docentes (Soto Hernández, 2016). Para Jogan y Johnson (2016), es necesario que los docentes de educación superior comprendan las motivaciones y características propias de las nuevas generaciones de estudiantes, como estrategia para relacionarse con ellos en el aula. Además, estos docentes debiesen tener competencias asociadas al liderazgo transformacional, para promover instancias de innovación y otros cambios coherentes con el escenario de la educación global (Gómez Barrios, 2018).

Favorecer el aprendizaje de todos los estudiantes en educación superior, para alcanzar las competencias comprometidas en los perfiles de egreso, es siempre un desafío de la gestión académica en las universidades (Bridgstock, 2016; Jollands, 2015), especialmente con los cambios observados en la población universitaria. Actualmente, los centros de desarrollo docente han proliferado y se han profesionalizado al interior de las instituciones; incorporándose metodologías constructivistas del aprendizaje al interior de las aulas y capacitándose a los docentes en estrategias de enseñanza activas. De hecho, según una investigación cualitativa de Calderón Flández (2018), en la que se entrevistó a 
docentes de medicina de la Universidad de Chile y fonoaudiología de la Universidad de Valparaíso, este escenario de mayor diversidad hizo que los docentes universitarios desarrollen concepciones de docencia que derivan en estrategias pedagógicas orientadas a la puesta en práctica del conocimiento. Esto último permite que, las experiencias de aprendizaje y los contenidos se vuelvan más homogéneos dentro del curso. Un ejemplo de metodología implementada en las aulas del país, es el método de estudio de casos (Valderrama, Azócar, de Bruijn, y González, 2016).

Este tipo de metodología abre las puertas a las innovaciones de tipo curricular, que, según reflexionan Labra Godoy et al. (2017), permite que la comunidad académica pueda observarse a sí misma y reflexionar acerca de sus acciones, para formar a los estudiantes de pregrado. Para lograr esto, se deben dejar atrás ciertas concepciones tradicionales y estáticas sobre el proceso de enseñanzaaprendizaje, entregando mayor protagonismo a los estudiantes como activos aprendices y a los docentes como los mediadores del aprendizaje. Sin embargo, a pesar de todos los cambios e innovaciones que se han visto en el último tiempo, aún falta rigurosidad a la hora evaluar el impacto $y$ efectividad de sus resultados.

Una de las metas de la educación superior es potenciar la capacidad de resolver problemas en contextos reales (Kalyuga, Renkl, y Pass, 2010). La experiencia tiene un papel crucial en el desarrollo de esta habilidad (Bernik y Žnidaršic, 2012), ya que es una fuente de conocimiento para analizar y resolver problemas a través de la combinación de la teoría y la práctica (Brickner y Etter, 2008; Hawtry, 2007). Sin embargo, como plantean Beard y Wilson (2002), si bien la experiencia puede sustentar el aprendizaje, este no puede darse por sentado. Se requiere que los estudiantes se involucren y reflexionen metódicamente sobre la experiencia, procesándola cognitiva y emocionalmente (Barnes, 2016).

Este trabajo muestra cómo el aprendizaje experiencial puede ser una valiosa metodología para enseñar competencias complejas, disciplinares y genéricas. Realizar actividades prácticas, en etapas tempranas de la carrera universitaria, donde los estudiantes deban desplegar un desempeño profesional, resulta enriquecedor y motivador en su formación académica. Además, los prepara para su futuro desempeño profesional de la forma más auténtica posible.

Las limitaciones de este trabajo tienen relación con su enfoque no experimental y la medición del cambio en relación al aprendizaje obtenido por los estudiantes. Sin embargo, el artículo permite conocer el modelo de aprendizaje experiencial, y comprender cómo puede aplicarse en la formación profesional, asimismo cómo se implementó en un curso, pudiendo ser un modelo para futuras innovaciones docentes en psicología u otras áreas. La experiencia fue valorada por docentes y estudiantes, con efectos positivos en el desempeño de los estudiantes y a la forma en que se vincularon con el contexto y su futuro rol como psicólogos. 


\section{Revisión de literatura}

En el aprendizaje experiencial, los estudiantes están involucrados y comprometidos, participando de forma activa en su proceso de aprendizaje (Munge, Thomas, y Heck, 2018). Se trata de un aprender haciendo (Blair, 2016; Seaman, Brown, y Quay, 2017) en un lugar y tiempo especificado (Smith y Segbers, 2018), en el que las interacciones y el contacto con las personas son clave porque involucran significados socioculturales e históricos (Harper, 2018). El compromiso con el lugar es imperativo, para que los participantes piensen de manera más profunda y crítica sobre las normas y estructuras sociales (Deringer, 2017), proporcionando una experiencia de vida más amplia (Ribbe, Cyrus, \& Langan, 2016).

$\begin{array}{ccccc}\text { Bailey, } & \text { Johhan } & \text { y } & \text { Kang } & (2017) \\ \text { plantean } & \text { que, } & \text { la } & \text { experiencia }\end{array}$ contextualizada facilita el proceso de inducir la disonancia cognitiva, ya que los estudiantes son desafiados y desestabilizados, por lo que se empujan los límites de lo que ellos conocen (McGowan, 2016). Por otra parte, también es necesaria la reflexión crítica sobre la experiencia, ya que actúa como mediadora de la construcción de significado. La naturaleza compleja de la resolución de problemas, relacionada con el aprendizaje experiencial, exige un pensamiento de orden superior (Collins, Sibthorp y Gookin, 2016), como el pensamiento crítico y prácticas de colaboración entre pares.

El aprendizaje experiencial es una potente herramienta para hacer ese cambio en la educación superior (Boud, Cohen, y Walker, 1993; Miettinen, 2000; Morris y Bilich-Eric, 2016). Esta metodología facilita que los estudiantes transfieran lo que han aprendido en clases hacia situaciones auténticas (Guo, Yao, Wang, Yang, y Zong, 2016), logrando una comprensión profunda de los contenidos (Baker, Jensen, y Kolb, 2005; Kayes, Kayes, y Kolb, 2005). Esta forma de aprender involucra dotar al estudiante de mayor autonomía y responsabilidad, implicándolo personalmente con su proceso de aprendizaje y en el contexto en que se desarrolla (Boud y Miller, 1996).

Respecto a sus beneficios, el aprendizaje experiencial ayuda a los estudiantes a establecer conexiones entre la experiencia y la teoría (Earnest, Rosenbusch, Wallace-Williams y Keim, 2016; Romero, 2010), facilitando la transición entre la educación de pregrado y mundo laboral (Friedman y Goldbaum, 2016). Además, debido a la utilización de distintos modos de aprendizaje (abstracto, concreto, reflexivo y activo), se profundiza en los contenidos, logrando un aprendizaje significativo (Calvert, Crowe, y Grenyer, 2016). Este artículo refiere a un estudio de caso realizado en la asignatura de psicología de la adultez con estudiantes de segundo año de la carrera de psicología.

Fases y modos del aprendizaje experiencial para aprender haciendo

El aprendizaje experiencial involucra la adquisición de conocimientos a través de la captación y transformación de la 
experiencia concreta, que se refleja en nuevas abstracciones y aplicaciones de la misma (Kolb, 1984; Kolb, Boyatzis, y Mainemelis, 2001). A partir de ello, este aprendizaje produce cambios, a nivel cognitivo, conductual y actitudinal en los estudiantes (Cayne, 2014; Illeris, 2007; Wright, 2000; Yeo y Marquardt, 2015). Ofrece a los estudiantes la oportunidad de practicar los conocimientos adquiridos en una situación real, modelando los comportamientos y procedimientos apropiados para desempeñarse en ésta (Knobloch, 2003).

A través de esta metodología, los estudiantes van más allá de la memorización, avanzando hacia el análisis y aplicación de lo aprendido, reflexionando respecto a las condiciones en que la aplicación de su conocimiento es útil (Bransford, Brown, y Cocking, 2000; Zelechoski, Riggs, y Wolbransky, 2017). El estudiante cumple un rol activo en la construcción del conocimiento

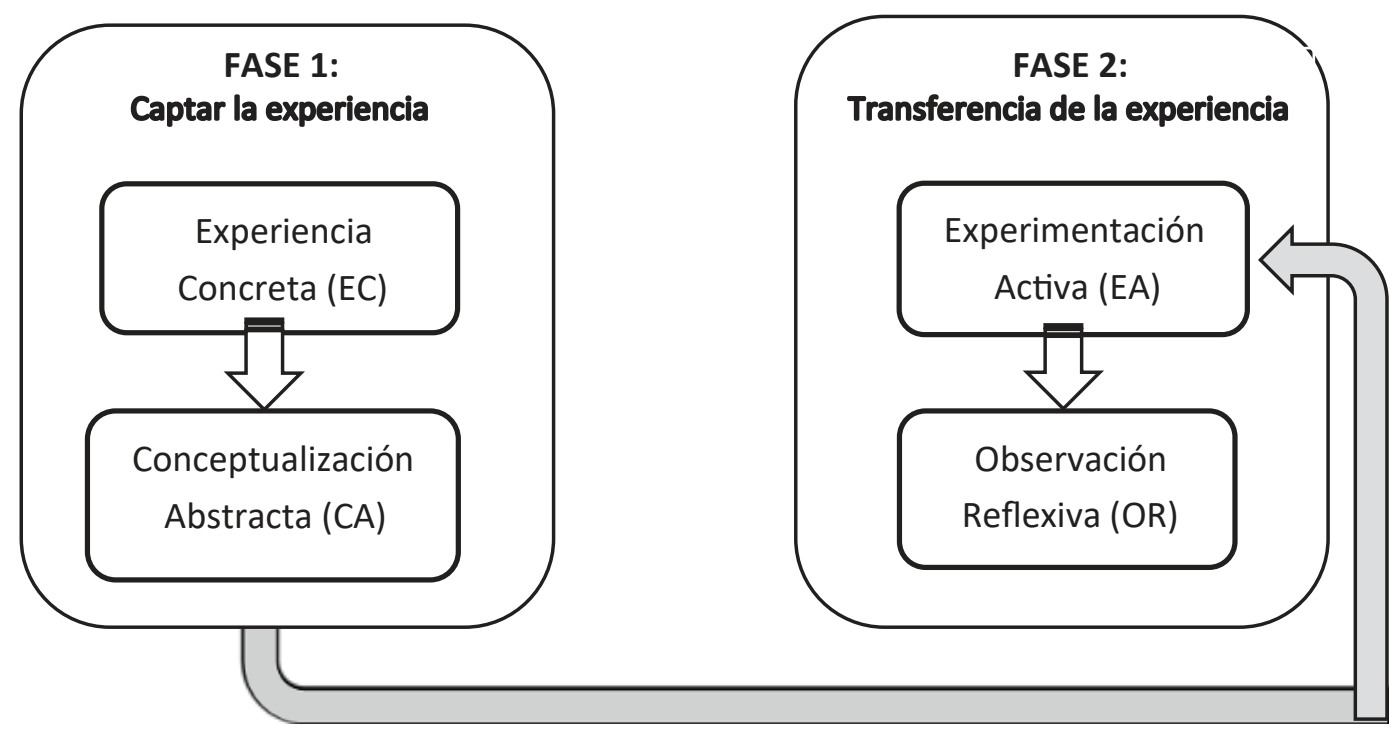

Figura 1. Modelo de aprendizaje experiencial.

Fuente: Dewar \& Walker (1999); Kolb (1981; 1984).
(Boud, Keogh, y Walker, 1985) a través de la aplicación situada del saber (Newns, Paul, y Creedy, 2015), y la traducción del conocimiento implícito en explícito (Abdulwahed y Nagy, 2009).

El aprendizaje experiencial involucra el tránsito por cuatro modos de aprendizaje, que ocurren dentro de dos fases. Existe un proceso dialéctico al interior de cada fase $\mathrm{y}$ entre estas. Asimismo, al interior de cada una existe un conflicto cognitivo, que demanda ajuste $y$ adaptación por parte del estudiante. En la primera fase se pone en juego lo concreto y abstracto, pasando desde la observación de una situación auténtica y abierta, hasta la conceptualización de un fenómeno que entrega un foco al proceso experiencial. En la segunda fase, interactúan procesos cognitivos prácticos $\mathrm{y}$ reflexivos, experimentando y luego reflexionando sobre la acción (Dewar y Walker, 1999; Kolb, 1981; 1984). La Figura 1 muestra este modelo. 
A continuación, se describen las dos fases de este modelo y los dos modos de aprendizaje dentro de cada fase. Se ilustra cada modo utilizando ejemplos de la adultez tardía, haciendo alusión al área de aplicación que será explicada más adelante.

\section{Fase 1: Captar la experiencia}

Se trata de una etapa en que los estudiantes deben llevar a cabo un proceso de pensamiento inductivo, pasando desde experiencias reales, concretas y tangibles, a la interpretación y teorización de ellas, convirtiéndolas en experiencias abstractas. En la conceptualización se relaciona y profundiza lo vivido, integrando contenidos para complejizar su análisis (Kolb, 1981; 1984). Los dos modos que componen esta fase son los siguientes:

Experiencia concreta (EC): implica experienciar, a través de la propia implicación personal, una situación concreta "contextualmente rica" a nivel del ambiente (incluidos los aspectos comunitarios, culturales y sociales) y el tiempo (presente y/o histórico). Se hace uso de la observación, la lectura de registros auténticos, y la conversación informal, desde donde surge una pregunta o un problema que interesa conocer (Calvert et al., 2016). Por ejemplo, la experiencia de visitar la plaza de un pueblo o un hogar de ancianos, teniendo la oportunidad de observar y conversar con adultos mayores y sus cuidadores, participar del encuentro con sus familias, puede llevar a un interés cognitivo y emocional sobre un tema en particular ligado la tercera edad. La experiencia se puede registrar a modo de notas, dibujos y/o pensamientos en un cuaderno de notas de campo.

Conceptualización abstracta (CA): supone darle sentido a la experiencia de forma abstracta, al examinar distintos medios simbólicos que se refieren a ella. Las conceptualizaciones abstractas pueden interpretarse críticamente, siendo específicas al contexto en estudio. Por ejemplo, revisar artículos científicos sobre el desarrollo humano en la tercera edad, columnas de opinión en los diarios, películas y documentales, y/o páginas web o blogs referidos al tema. Es decir, a partir del estímulo de la experiencia concreta, se conceptualiza un fenómeno apoyado por el análisis que otros sociales han realizado. Luego, se integra la experiencia personal con las opiniones, expresiones y simbolizaciones sobre el tema en un producto que puede ser una columna de opinión.

\section{Fase 2: Transformar la experiencia}

En esta etapa, los estudiantes deben desplegar un proceso de pensamiento deductivo, en el cual se mueven desde la acción en el mundo concreto hacia la reflexión abstracta (Kolb, 1981; 1984). Los dos modos que componen esta fase son los siguientes:

Experimentación activa (EA): enfatiza aplicación práctica e implica que los estudiantes vuelven al "terreno" o la realidad, para profundizar en su búsqueda de comprensión de un tema, o bien, a probar teorías. Se trata de examinar la 
adecuación de las conceptualizaciones abstractas formuladas previamente, frente a nuevas experiencias concretas. El aprendizaje experiencial es un proceso que aparta, de forma deliberada, a los estudiantes de sus zonas de confort y, en consecuencia, pueden aprender a apreciar que las condiciones cambian, a veces de forma muy discreta, a través del tiempo y el lugar. A partir de la primera fase, se logra empatizar y comprender un fenómeno desde la propia experiencia y la de otros, pero falta contrastar esta construcción con la realidad, es decir, volver a ella. Por ejemplo, los estudiantes realizan un estudio en profundidad sobre la tercera edad, construyen y aplican una entrevista al adulto mayor, donde indagan sobre su experiencia de vida y el cumplimiento de las tares del ciclo vital, según la etapa y contexto en que se encuentran.
Observación reflexiva (OR): se busca la comprensión del fenómeno estudiado, como también, se concluye en relación a cómo las etapas anteriores facilitaron o limitaron este entendimiento (Calvert et al., 2016). En el proceso, los alumnos deben actuar como un investigador $\mathrm{y}$ analizar la idoneidad de nuevos o preexistentes conceptualizaciones abstractas contra la experiencia del mundo real (Hagan, 2012). Por ejemplo, hacer un análisis de la entrevista y una reflexión personal donde se integren cada una de las etapas anteriores y la vivencia personal de profundizar en la etapa evolutiva de la adultez mayor. El producto de esta etapa puede ser la presentación en un poster del trabajo realizado y la defensa oral de los hallazgos y conclusiones realizadas. La Tabla 1 muestra la síntesis de las fases y modos antes señalados.

Tabla 1.

Síntesis de fases y modos de aprendizaje experiencial

\begin{tabular}{|c|c|c|c|}
\hline Fases & Modos & Productos & Función \\
\hline $\begin{array}{l}\text { Fase 1: } \\
\text { Captar la } \\
\text { experiencia }\end{array}$ & $\begin{array}{l}\text { Conceptualización } \\
\text { abstracta }(\mathrm{CA})\end{array}$ & $\begin{array}{l}\text { Cuaderno de } \\
\text { notas de } \\
\text { campo. }\end{array}$ & $\begin{array}{l}\text { Implicarse y experienciar, a nivel } \\
\text { cognitivo y emocional, una } \\
\text { situación concreta, contextualmente } \\
\text { rica, en un lugar ytiempo determinado. } \\
\text { Dar sentido a la experiencia de } \\
\text { forma abstracta a partir de distintos } \\
\text { medios simbólicos que otros han } \\
\text { construido del fenómeno de interés. }\end{array}$ \\
\hline $\begin{array}{l}\text { Fase 2: } \\
\text { Transformar la } \\
\text { experiencia }\end{array}$ & $\begin{array}{l}\text { Experimentación } \\
\text { activa (EA) }\end{array}$ & $\begin{array}{l}\text { Estudio en } \\
\text { profundidad }\end{array}$ & $\begin{array}{l}\text { Volver al "terreno" o la realidad, } \\
\text { para profundizar y testear/ contrastar } \\
\text { conceptualizaciones construidas a } \\
\text { partir de la experiencia y la revisión } \\
\text { de materiales y documentos. } \\
\text { Concluir y evaluar la comprensión } \\
\text { del fenómeno, analizando la } \\
\text { idoneidad, calidad, sentido, } \\
\text { función, desafíos y consecuencias } \\
\text { del nuevo entendimiento alcanzado. }\end{array}$ \\
\hline
\end{tabular}

Fuente: Elaboración propia. 
Lo óptimo en el aprendizaje experiencial es lograr un balance adecuado entre estos cuatro modos del aprendizaje para abordar en profundidad un fenómeno. El aprendiz debe transitar por todos ellos (experienciar y pensar, actuar y reflexionar), de manera recursiva, en base a la situación $\mathrm{y}$ contenidos de aprendizaje. El proceso de aprendizaje implica que el estudiante se mueva gradualmente de actor a observador, y desde la participación activa al análisis global (Itin, 1999).

Por último, es importante dedicar un apartado al Design Thinking, el cual es un proceso delimitado por una serie de pasos, que puede ser usado tanto por diseñadores como no diseñadores. El primer paso, llamado "Empatizar", consiste en conocer cuál es el problema, lo cual implica mantener una conversación y pasar tiempo con quienes se encuentran afectados por éste. Definir es el segundo paso, en el que toda la información recopilada anteriormente es usada para delimitar el desafío. A continuación, está el paso "Idear", en el que se proponen diferentes propuestas de solución al problema; se puede buscar inspiración de cualquier lugar $\mathrm{y}$ co-diseñar con distintas personas. El paso siguiente es "Prototipar" que requiere que se prueben las soluciones propuestas en pequeña escala, para decidir cuáles sirven y cuáles no. Finalmente, está el paso de "Evaluar", donde se juzga cuán pertinente es la solución al problema detectado. Los tres primeros pasos consisten en poner en práctica el pensamiento divergente, al explorar un problema, y los dos últimos en enfocar las acciones a través del pensamiento convergente (Design Council, 2015).

Se ha encontrado que esta metodología es útil en el ámbito empresarial y educacional, ya que entrega un marco de referencia para la resolución de problemas, que contempla enfoques analíticos y creativos (Liedtka, 2017). No obstante, también se puede aplicar en el ámbito de la educación superior. Badwan, Bothara, Latijnhouwers, Smithies y Sandars (2017) sostienen que el Design Thinking permite a estudiantes de medicina abordar problemas complejos de manera creativa e innovadora y, aprender habilidades de aprendizaje transferibles a lo largo de la vida para enfrentar la incertidumbre y el trabajo en equipo. Por lo tanto, esta puede ser aplicada en distintos ámbitos de la educación médica, como en proyectos de intervención tecnológica e innovación curricular.

\section{Metodología}

La implementación de esta metodología se realizó en la asignatura de Psicología evolutiva en la adultez, que se dicta en el cuarto semestre de la carrera de psicología, siendo la tercera asignatura y final del área evolutiva (la primera está relacionada con la primera infancia y la segunda con infancia y adolescencia). Es un estudio no experimental donde participaron 3 docentes y 3 ayudantes de la asignatura, y se realizó durante 14 semanas para 90 estudiantes (3 grupos de clases), trabajando de manera constante en las instancias de ayudantía y con una presentación del trabajo realizado hacia 
el final del semestre, a modo de cierre del proceso. Este artículo puede definirse como un estudio de caso desarrollado en una asignatura de la carrera de psicología en educación superior.

En el método de estudio de caso los datos son obtenidos desde una variedad de fuentes, tanto cualitativas como cuantitativas; esto es, documentos, registros de archivos, entrevistas directas, observación directa, observación de los participantes e instalaciones u objetos físicos. Es un método de estudio de caso apropiado para estudios exploratorios desarrollados en su entorno real (Martínez, 2006). En este estudio en particular, se consideraron los productos que elaboraron los estudiantes (obtenidos de cada etapa de evaluación), la observación directa de estos en clases y en terreno, además de sus calificaciones, comparadas con las del curso del año anterior.

Es importante considerar el perfil de egreso de la carrera de psicología para comprender la importancia de incorporar esta metodología. El perfil contiene competencias específicas, organizadas en Dominio Teórico, Integración Sistémica, Rigurosidad Científica, Diagnóstico e Intervención. Además, contempla el desarrollo de ocho competencias genéricas, las cuales son Eficiencia, Responsabilidad Pública, Ética, Autonomía, Emprendimiento y Liderazgo, Visión Global, Comunicación y Visión Analítica. Esta asignatura en particular se encuentra alineada con lo declarado en el perfil egreso, contribuyendo específicamente a la competencia genérica de Responsabilidad Pública y a la competencia específica de Diagnóstico, ambas a nivel de Bachillerato.

Respecto a la competencia genérica de Responsabilidad Pública, se declara que el estudiantado debe lograr:

-Identificar los aportes de la disciplina para el mejoramiento de la calidad de vida de las personas, a lo largo del ciclo vital, en iniciativas de instituciones públicas y privadas.

-Investigar sobre los problemas psicosociales, que se presentan en nuestro país, mostrando interés en su solución.

De acuerdo a la competencia específica de Diagnóstico, los estudiantes deben lograr:

-Evaluar necesidades, a partir del uso de técnicas de observación y entrevista, respetando las etapas de un proceso de diagnóstico.

El objetivo de esta asignatura es que los estudiantes puedan analizar el desarrollo humano durante la adultez, integrando las etapas previas $y$ discutiendo diversas teorías y modelos que lo han abordado. Lo anterior se realiza desde una mirada relacional y situada que permite comprender el desarrollo humano dentro de un contexto social e interpersonal.

La incorporación de la metodología del aprendizaje experiencial, tuvo como objetivo promover que los estudiantes pudieran aplicar y practicar conocimientos en una situación real y 
contextualizada, y que esto tributara a su aprendizaje profundo. Para ello, se planteó a los estudiantes trabajar con las etapas del ciclo vital, para que pudiesen conocer distintas áreas del desarrollo, tales como físico, socioemocional, cognitivo, moral y de personalidad.

Los estudiantes se organizaron en grupos de 4 a 5 personas, para realizar una observación en un contexto natural que les permitiera escoger una etapa del ciclo vital y una problemática asociada a ésta, para posteriormente, seleccionar diferentes instituciones en las que pudieran acceder a personas de dicho rango etario.

\section{Resultados}

A continuación, se describe la aplicación del aprendizaje experiencial, considerando cada una de sus fases y modos, en la asignatura de psicología de la adultez.

Fase 1: Captar la experiencia.

1.1.- Experiencia Concreta (EC). Los estudiantes conformaron grupos $\mathrm{y}$ escogieron una etapa del ciclo vital que fuera de su interés: adultez joven, intermedia o mayor. Esto lo hicieron para decidir dónde realizar una observación directa en un contexto realista y libre, que permitiera observar e interactuar con sujetos que pertenecieran al grupo etario seleccionado. De esta forma, acudieron a espacios tales como plazas, supermercados y parques, entre otros. Esta instancia constituyó la primera aproximación al tema que escogieron, en las que se les pedía asumir un rol de observador participante, en el sentido que podían mantener conversaciones informales y realizar observaciones del contexto y las personas con quienes trabajarían. Se les planteó la importancia de asistir sin ideas preconcebidas y registrar aquello que llamara su atención en una bitácora, para identificar una problemática que tendrían que desarrollar.

\section{2.- Conceptualización Abstracta} (CA). Una vez realizada la observación, los estudiantes sistematizaron la información e identificaron un área del desarrollo y problemática asociada que fuera de su interés. Posteriormente y, de manera individual, realizaron un ensayo breve acerca de lo anterior, para reflexionar en torno a las problemáticas que habían observado, analizando de manera empírica y teórica. Para esto, revisaron bibliografía y artículos científicos pertinentes según el tema escogido y entregaron el ensayo como producto, el que fue revisado $y$ retroalimentado en instancias de ayudantía.

\section{Fase 2: Transformar la Experiencia}

2.1.- Experimentación activa (EA). En esta etapa, los estudiantes asistieron a instituciones en las cuales había personas de la etapa del ciclo vital estudiado y la problemática escogida. Para ello, se solicitó ayuda de parte del Servicio de Psicología Integral (SPI) de la Universidad con la gestión del contacto. Una vez en la institución, los grupos procedieron a realizar entrevistas que les 
permitieron recabar información $\mathrm{y}$ empatizar con las personas, para generar un producto o solución mediante la metodología Design Thinking, que considera cinco etapas: Empatizar, Definir, Idear, Prototipar y testear. Los estudiantes diseñaron instrumentos de recolección de datos (entrevistas y observaciones) y a partir de esta información, definieron el problema que se pretendía solucionar, planteándose preguntas tales como ¿a quién le afecta el problema?, ¿dónde ocurre el problema?, ¿de qué se trata el problema?, ¿qué provoca? Una vez definido el problema a abordar, realizaron una lluvia de ideas sobre posibles soluciones que pudieran responder a esta necesidad y volvieron a la institución a preguntar si estas soluciones parecían solucionar el problema y eran plausibles de implementar.

2.2.- Observación Reflexiva (OR). Tomando la información obtenida en las etapas anteriores, los estudiantes integraron ésta con la información teórica revisada. A partir de lo anterior, eligieron la solución más adecuada para el problema detectado, de acuerdo a las características de la etapa evolutiva y los desafíos que enfrenta la población escogida en esa área del desarrollo en particular. Más tarde, los estudiantes materializaron sus soluciones en prototipos.

Cada prototipo fue presentado por el grupo durante las últimas clases del semestre, ante una comisión conformada por las docentes del curso y un docente externo a la asignatura, con el fin de que pudieran justificar teórica $\mathrm{y}$ prácticamente la utilidad de ésta para responder a la problemática planteada. Esto se realizó desde la lógica de "Elevator Pitch", el cual corresponde a un discurso convincente de presentación en el que el estudiante debe condensar un mensaje que llame la atención de los miembros de la comisión en muy pocos minutos. Para finalizar, como grupo reflexionaron acerca del proceso de trabajo y cómo esto les permitió integrar los contenidos teóricos revisados y la experiencia concreta como modo de aprendizaje.

Cabe destacar que todos los productos que elaboraron los estudiantes coinciden con los que están indicados en la Tabla 1 (ver en Revisión de literatura), cumpliéndose a cabalidad lo planificado de acuerdo a las competencias genéricas.

Desde la competencia genérica de Responsabilidad Pública, se esperó que los estudiantes pudieran identificar los aportes de la disciplina para el mejoramiento de la calidad de vida de las personas, a lo largo del ciclo vital, en iniciativas de instituciones públicas y privadas. Es posible decir que esto fue logrado, ya que el proyecto se centró en identificar necesidades propias de personas que se encontraran cursando la adultez temprana, media o mayor $\mathrm{y}$ desarrollar soluciones a estas mediante los contenidos del curso y la revisión de literatura asociada. Esto se realizó a lo largo de todas las etapas, mientras que el trabajo directo con instituciones de tipo público y privado fue desde la etapa de Experimentación Activa. El vínculo con 
estas instituciones tributa directamente a la competencia genérica, con el fin de que las soluciones creadas por los estudiantes impactaran en ellas.

De acuerdo a la misma competencia genérica anterior, se esperó que los estudiantes investigaran sobre los problemas psicosociales que se presentan en nuestro país, mostrando interés en su solución. Esto también se cumplió, incluso en la primera fase, en la que observaron e identificaron un tema (Experiencia Concreta), para investigar más sobre este posteriormente (Conceptualización Abstracta). De hecho, la evaluación final del curso, en la etapa de Observación Reflexiva, consistió en la presentación de la solución que construyeron.

Por otro lado, desde la competencia de Diagnóstico, los estudiantes detectaron necesidades a partir del uso de técnicas de observación y entrevista, respetando las etapas de un proceso de diagnóstico. La técnica de observación fue usada en la etapa de Experiencia Concreta, mientras que la entrevista en la etapa de Experimentación Activa. De no haber realizado un adecuado proceso de diagnóstico, los estudiantes no hubiesen podido construir un prototipo de solución satisfactorio, adecuado a la etapa evolutiva y contexto que investigaron.

En este curso, correspondía evaluar a los adultos en distintas áreas de su desarrollo y su calidad de vida, detectando necesidades y áreas de mejora relevantes de considerar en el trabajo del psicólogo y el perfeccionamiento de las políticas públicas del país, tomando en consideración los modelos teóricos-metodológicos y los elementos del contexto que resultan pertinentes. Esto está en directa relación con las competencias abordadas anteriormente, aunque también hace mención explícita a las políticas públicas del país. La evaluación descrita en la competencia fue desarrollada a lo largo del semestre, a medida que los estudiantes cumplían con las etapas y productos solicitados.

Es importante destacar que, como cada contenido de la asignatura está asociado a un Resultado de Aprendizaje, el cual al mismo tiempo está relacionado con la competencia genérica o específica del curso, el diseño de instrumentos de evaluación se hace más sencillo, ya que lo que se debe medir está explicitado. Además, los estudiantes también saben lo que se espera de ellos, por lo que pueden prepararse de mejor manera para sus evaluaciones y así lograr el desempeño esperado.

Por último, las calificaciones de los estudiantes en esta actividad estuvieron sobre el promedio de las notas anteriores, con una diferencia significativa entre ellas $(p<0.05)$. Por otra parte, en sus reflexiones finales, los estudiantes consistentemente valoraron la experiencia, plantearon que se sintieron desarrollando un rol profesional y que aprendieron más que en otras actividades académicas tradicionales.

\section{Conclusiones}

Si bien los estudiantes de educación 
superior comienzan su formación a través de la lectura de textos y clases dentro del aula, profundizando de manera teórica y conceptual sobre los temas tratados, en algún momento deberán aplicar sus conocimientos en el mundo real. Sin embargo, el tránsito desde aprender en la sala de clases hacia la aplicación en la realidad, tiende a no ser suficientemente "andamiada" a través de metodologías de enseñanza y aprendizaje pertinentes y coherentes. Es por esto que, alternativas como la metodología de aprendizaje experiencial ofrecen espacios para que los estudiantes puedan aplicar su saber en una situación auténtica (Guo et al., 2016), una especie de zona intermedia entre la teoría y el desempeño profesional sin supervisión, que se espera que puedan ejecutar una vez egresados. En otras palabras, facilitan la transición entre pregrado y mundo laboral, tal y como plantean Friedman y Goldbaum (2016).

En el caso de la presente experiencia, los estudiantes tuvieron un acercamiento gradual al mundo real, de la mano con los contenidos del curso. Comenzaron, de manera grupal, con la observación directa de adultos (la elección de la etapa del ciclo vital dependía del interés de los estudiantes) en lugares públicos (Experiencia Concreta), para después sistematizar la información e identificar una problemática. A partir de esta actividad $y$ de una revisión de bibliografía, elaboraron un ensayo para reflexionar sobre la temática (Conceptualización Abstracta). Estos pasos conformaron la Fase 1 (Captar la experiencia) de la teoría propuesta por Kolb (1981; 1984). La Fase 2
(Transformar la Experiencia) consistió en que los estudiantes asistieran a instituciones en las que pudiesen investigar acerca de la temática que escogieron, buscar posibles soluciones a través de la metodología Design Thinking (Experimentación Activa) y elaborar un prototipo de solución con la idea seleccionada. Este último fue presentado ante una comisión como evaluación final (Observación Reflexiva). A partir de lo descrito, es posible afirmar que el Design Thinking es una metodología compatible con el aprendizaje experiencial y aplicable en educación superior para abordar problemas profesionalizantes, no solo en educación médica (Badwan et al., 2017), sino que en otras disciplinas como la psicología.

Cabe destacar que, estas actividades se desarrollaron en el cuarto semestre de la carrera de psicología, de un total de diez. Entonces, si los estudiantes ya están realizando este tipo de tareas en su segundo año, posiblemente tendrán más facilidades para adaptarse y tener un buen desempeño en actividades prácticas y en terreno en cursos superiores. Por lo tanto, es necesario destacar la relevancia que los estudiantes puedan aprender a aplicar sus conocimientos en contextos realistas, en etapas tempranas de su formación. El aprendizaje experiencial, justamente lo permite (Knobloch, 2003). También, entrega herramientas a los docentes para desplegar sus competencias de liderazgo transformacional (Gómez Barrios, 2018) $\mathrm{y}$ que conozcan las motivaciones e intereses de sus estudiantes, cumpliendo con la propuesta de Jogan y Johnson (2016), ya que los estudiantes no solo 
siguieron instrucciones, sino que tuvieron un amplio espacio para tomar decisiones de acuerdo a sus intereses, eligiendo dónde y con quiénes trabajar.

Por otro lado, otra fortaleza del estudio fue que la experiencia fue rigurosamente planificada, dirigida $\mathrm{y}$ monitoreada por docentes y ayudantes, siguiendo los lineamientos institucionales de la Universidad y de la Facultad. Contó con objetivos, etapas y actividades de evaluación, claramente delimitadas $\mathrm{y}$ pensadas para que los estudiantes pudiesen transformar su conocimiento desde lo concreto a lo abstracto, mirarlo desde distintos ángulos y formatos, pero con un anclaje en la realidad. Específicamente en este caso, los estudiantes pudieron observar y acercarse a problemáticas relacionadas con distintas etapas del ciclo vital, especialmente en las etapas de Experiencia Concreta y Experimentación Activa. Adicionalmente, pudieron reflexionar, analizar y vincular estas experiencias con la teoría y literatura científica en las etapas de Conceptualización Abstracta y Observación Reflexiva. De esta forma, a través de la experiencia auténtica, los estudiantes combinaron la teoría y la práctica para resolver una problemática (Brickner y Etter, 2008; Hawtry, 2007).

No obstante, para que una experiencia sea exitosa no basta solamente con una buena planificación y ejecución. La evaluación focalizada del desempeño de los estudiantes en cada una de las etapas es necesaria para poder hacer un seguimiento de la experiencia de estos.
¿Están cumpliendo con los objetivos propuestos? ¿Cuáles son sus inquietudes, dudas y conclusiones? ¿Están leyendo la literatura adecuada para poder comprender las temáticas y fenómenos que escogieron? Sin duda, para poder responder estas preguntas se necesita una adecuada evaluación formativa $\mathrm{y}$ sumativa, la que debe ser coherente con los lineamientos institucionales y con los objetivos y resultados de aprendizaje de la asignatura. Tal y como concluyen Bernate, Guataquira Romero, Romero Melo y Reyes Escobar (2020), la medición de los procesos educativos son imperantes para la mejora de las condiciones de los programas.

La aplicación de la teoría de Kolb (1981; 1984) llevada a cabo, demuestra que no es necesario esperar a que los estudiantes de una carrera universitaria alcancen los últimos cursos del programa para poder desempeñarse "en terreno". Estas experiencias deben planificarse, ejecutarse y evaluarse con rigurosidad, siguiendo el modelo teórico de Kolb, para así lograr el máximo aprendizaje de los estudiantes y al mismo tiempo cumplir con todos los criterios éticos de intervención, sin poner en riesgo el bienestar de los estudiantes ni de las otras personas que se encuentran participando en el proceso. La evaluación es especialmente relevante para detectar oportunidades de mejora y perfeccionar futuras experiencias.

\section{Contribución de los autores}

V.A.V.H. Idea, revisión de literatura, y redacción de conclusiones. 
M.P.G.S. Idea, metodología, y discusión de resultados.

D.V.B.J. Idea y discusión de resultados.

I.F.C.R. Revisión de literatura, y redacción de conclusiones.

\section{Referencias}

Abdulwahed, M., y Nagy, Z. (2009). Applying Kolb's experiential learning cycle for laboratory education. Journal of Engineering Education, 98(3), 283-294. doi:10.1002/j.2168-9830.2009.tb01025.x

Araneda-Guirriman, C., y Pedraja-Rejas, L. (2017). Las universidades y el nuevo perfil de estudiantes: nuevas realidades y retos para América Latina. Interciencia, 42(12), 786-788

Badwan, B., Bothara, R., Latijnhouwers, M., Smithies, A., y Sandars, J. (2017). The importance of design thinking in medical education. Medical Teacher, 40(4), 425-426. doi:10.1080/0142159X.2017.13 99203

Bailey, A. W., Johann, J., y Kang, H. K. (2017). Cognitive and physiological impacts of adventure activities: Beyond self-report data. Journal of Experiential Education, 40(2), 153-169. doi:10.1177/1053825917 701250

Baker, A.C., Jensen, P.J., y Kolb, D.A. (2005). Conversation as experiential learning. Management Learning, 36(4), 411-427. doi:10.1177/1350507605058130

Barnes, M. E. (2016). The student as teacher educator in service-learning. Journal of Experiential Education, 39(3), 238-253. doi:10.1177/1053825916643831

Beard, C., y Wilson, J. P. (2002). The power of experiential learning: A handbook for trainers and educators. Kogan Page Limited.

Bernate, J.A., Guataquira Romero, A., Romero Melo, E.N., y Reyes Escobar, P.C. (2020). Satisfacción de la Calidad Educativa en Educación Superior. Podium, 38, 37-50 doi:10.31095/podium.2020.38.3

Bernik, M., y Žnidaršič, J. (2012). Solving complex problems with help of experiential learning. Organizacija, 45(3), 117-124. doi:10.2478/v10051-012-0012-6

Blair, D. J. (2016). Experiential learning for teacher professional development at historic sites. Journal of Experiential Education, 39(2), 130-144. doi:10.1177/ 1053825916629164

Boud, D., Cohen, R. y Walker, D. (1993). Using Experience for Learning. England: Open University Press.

Boud, D., Keogh, R., y Walker, D. (1985). Reflection: Turning experience into learning. United States of America: Nichols Publishing Company.

Boud, D., y Miller, N. (1996). Working with experience. Animating learning. Routledge

Bransford, J. D., Brown, A. L., y Cocking, R.R. (2000). How people learn? Brain, mind, experience, and school. United States of America: National Academy Press.

Brickner, D. R., y Etter, E. R. (2008). Strategies for promoting active learning in a principle of accounting course. Academy of Education Leadership Journal, 12(2), 87-93.

Bridgstock, R. S. (2016). The university and the knowledge network: a new educational model for 21 st century learning and employability. In M. Tomlinson (Ed.). Graduate employabiity in context: Research, theory and debate. United 
Kingdom: Palgrave Macmillan.

Calderón Flández, C. (2018). Concepciones educativas sobre la enseñanza y el aprendizaje en docentes universitarios en contextos de diversidad educativa. Innovare. Revista Electrónica de Educación Superior, 2(4), 66-87.

Calvert, F., Crowe, T., y Grenyer, B. (2016). Dialogical reflexivity in supervision: An experiential learning process for enhancing and relational competencies. Journal of supervision in psychotherapy and mental health, 35(1), 1-21. doi:10.1080/07325223.2015.1135840

Cayne, J. (2014). Learning beyond the known. European Journal of Psychotherapy \& Counselling, 16(3), 212-227. doi:10.10 80/13642537.2014.932298

Collins, R. H., Sibthorp, J., y Gookin, J. (2016). Developing ill-structured problemsolving skills through wilderness education. Journal of Experiential Education, 39(2), 179-195. doi:10.1177/ 1053825916639611

Deringer, S. A. (2017). Mindful place-based education: Mapping the literature. Journal of Experiential Education, 40(4), 333-348. doi:10.1177/1053825917716694

Design Council. (2015). What is the framework for innovation? Design Council's evolved Double Diamond. Retreived from https://www.designcouncil.org.uk/newsopinion/what-framework-innovation-desi gn-councils-evolved-double-diamond

Dewar, B., y Walker, E. (1999). Experiential learning: Issues for supervision. Journal of Advanced Nursing , 30(6), 1459-1467. doi:10.1046/j.1365-2648.1999.01228.x

Earnest, D., Rosenbusch, K., Wallace-Williams, D., y Keim, A. (2016). Study abroad in psychology: Increasing cultural competencies through experiential learning. Teaching of Psychology, 43(1) 75-79. doi:10.1177/0098628315620889

Friedman, F., y Goldbaum, C. (2016). Experiential learning: Developing insights about working with older adults. Clinical Social Work Journal, 44(2), 186-197. doi:10.1007/s10615-016-0583-4

Gómez Barrios, S. A. (2018). Tendencias globales en la educación universitaria: Una mirada desde el liderazgo transformacional. Podium, 33, 69-78. doi:10.31095/po dium.2018.33.7

Guo, F., Yao, M., Wang, C., Yang, W., y Zong, X. (2016). The effects of service learning on student problem solving: the mediating role of classroom engagement. Teaching of Psychology, 43(1), 16-21. doi:10. 1177/0098628315620064

Hagan, L. M. (2012). Fostering experiential learning and service through client projects in graduate business courses offered online. American Journal of Business Education, 5(5), 623-632. doi:10.19030/ajbe.v5i5.7220

Harper, N. J. (2018). Locating self in place during a study abroad experience: Emerging adults, global awareness, and the Andes. Journal of Experiential Education, 41(3), 295-311. doi:10.1177/1053825918761995

Hawtry, K. (2007). Using experiential learning techniques. The Journal of Economic Education, 38(2), 143-152. https://doi. org/10.3200/JECE.38.2.143-152

Illeris, K. (2007). What do we actually mean by experiential learning? Human Resource Development Review, 6(1), 84-95. doi:10.1177/1534484306296828

Itin, C. (1999). Reasserting the philosophy of experiential education as a vehicle for change in the 21st century. Journal of Experiential Education, 22(2), 91-98. doi:10.1177/105382599902200206 
Jogan, K. S., y Johnson, K. (2016). Estrategias educativas para el estudiante universitario de hoy. Innovare. Revista Electrónica de Educación Superior, 1(1), 27-38.

Jollands, M. (2015). A framework for graduate employability adapted for discipline differences. In Proceedings of the Higher Education Research and Development Society of Australia Conference (HERSA 2015), Melbourne, Australia, 6-9 July.

Kalyuga, S., Renkl, A., y Pass, F. (2010). Facilitating flexible problem solving: a cognitive load perspective. Educational Psychology Review, 22, 175-186. doi:10.1007/s10 648-010-9132-9

Kayes, A.B., Kayes, D.C., y Kolb, D.A. (2005). Experiential learning in teams. Simulation \& Gaming, 36(3), 330-354. doi:10.1177/1046878105279012

Knobloch, N. A. (2003). Is experiential learning authentic? Journal of Agricultural Education, 44(4), 22-34. doi:10.5032/ JAE.2003.04022

Kolb, D. A. (1981). Experiential learning theory and the learning style inventory: A reply to Freedman and Stumpf. Academy of Management Review, 6(2), 289-296. doi:10.5465/amr.1981.4287844

Kolb, D. (1984). Experiential learning: experience as a source of learning and development. United States of America: Prentice Hall.

Kolb, D. A., Boyatzis, R.E., y Mainemelis, C. (2001) Experiential Learning Theory: Previous Research and New Directions, in R.J. Sternberg and L. F. Zhang (eds) Perspectives on Thinking, Learning, and Cognitive Styles, pp.227-247. Mahwah, NJ: Lawrence Erlbaum.

Košutić, I. (2017). The role of cultural capital in higher education access and institutional choice. Center for Educational Policy Studies Journal, 7(1), 149-169.
Labra Godoy, P., Garcés Bustamante, J., Vargas Martínez, L., Cortés Iturrieta, P., Urrutia Castro, M., Vega Guerrero, L., Lotito Rojas, A., Serrano Benti, C., Calvo Muñoz, C., Arancibia Araya, E., Vásquez Aqueveque, A., Bonilla Meneses, P., Álvarez Cortes, M., Rodríguez Urquisa, J., Páez Rivera, H., Fuentealba Fabio, F., López de Maturana Luna, S., Yagnam Abufón, F. y Gary Bufadel, A. (2016). Aprendizaje en educación superior. Una mirada sistémica a los sujetos que aprenden, en un contexto de innovación curricular. Innovare. Revista Electrónica de Educación Superior, 1(1), 39-65.

Liedtka, J. (2017). Evaluating the impact of Design Thinking in action. Academy of Management Proceedings, doi:10.5465/ AMBPP.2017.177

Martínez, P. (2006). El método de estudio de caso: estrategia metodológica de la investigación científica. Pensamiento \& Gestión, (20), 165-193.

McGowan, A. L. (2016). Impact of one-semester outdoor education programs on adolescent perceptions of self-authorship. Journal of Experiential Education, 39(4), 386-411. doi:10.1177/1053825916668902

Miettinen, R. (2000). The concept of experiential learning and John Dewey's theory of reflective thought and action. International Journal of Lifelong Education, 19(1), 54-72. doi:10.1080/026 013700293458

Morris, E., y Bilich-Eric, L. (2016). A framework to support experiential learning and psychological flexibility in supervision: SHAPE. Australian Psychologist, 52(2), 104-113. doi:10.1111/ap.12267

Munge, B., Thomas, G., y Heck, D. (2018). Outdoor fieldwork in higher education: Learning from multidisciplinary experience. Journal of Experiential Education, 41(1), 39-53. doi:10.1177/1053825917742165 
Newns, K., Paul, M., y Creedy, K. (2015). Action ethics: Using action method techniques to facilitate training on ethical practice. Journal of Family Therapy, 37(2), 246-258. doi:10.1111/1467-6427.12020

Romero, M. (2010). El Aprendizaje experiencial y las nuevas demandas formativas. Revista de Antropología Experimental, 10(8), 98-102.

Ribbe Jr, R., Cyrus, R., y Langan, E. (2016). Exploring the impact of an outdoor orientation program on adaptation to college. Journal of Experiential Education, 39(4), 355-369. doi:10.1177/ 1053825916668900

Seaman, J., Brown, M., y Quay, J. (2017). The evolution of experiential learning theory: Tracing lines of research in the JEE. Journal of Experiential Education, 40(4), 1-21. doi:10.1177/1053825916689268

Smith, H. A., y Segbers, T. (2018). The impact of transculturality on student experience of higher education. Journal of Experiential Education, 41(1), 75-89. doi:10.1177/ 1053825917750406

Soto Hernández, V. (2016). Innovaciones en la formación de estudiantes de pedagogía: El caso del convenio de desempeño de una universidad chilena. Innovare. Revista Electrónica de Educación Superior, 1(2), 43-71.

Valderrama, N., Azócar, G., de Bruijn, J. y González, M. E. (2016). Método de estudio de casos en la enseñanza universitaria de estadística; aplicación, enfoques y estrategias docentes. Revista Electrónica de Educación Superior, 1(2), 72-89.

Wright, M. (2000). Getting more out of less: The benefits of short-term experiential learning in undergraduate sociology courses. Teaching Sociology, 28(2), 116-126. doi:10.2307/1319259
Yeo, R., y Marquardt, J. (2015). Reinterpreting action, learning and experience: Integrating action learning and experiential learning. Human Resource Development Quarterly, 26(1), 81-107. doi:10.1002/hrdq.21199

Zelechoski, A., Riggs, C., y Wolbransky, M. (2017). Teaching psychology and law: an empirical evaluation of experiential learning. Teaching of Psychology, 44(3), 222-231. doi:10.1177/0098628317711316. 